УДК 622.276.53.054

\title{
ПНЕВМОКОМПЕНСАТОР ДЛЯ ПЛУНЖЕРНОГО НАСОСА С ПОГРУЖНЫМ ЛИНЕЙНЫМ ПРИВОДОМ
}

\author{
Уразаков Камил Рахматуллович', \\ urazakk@mail.ru
}

Тимашев Эдуард Олегович1, timashev@mail.ru

\author{
Абдуллин Наиль Ахиярович1, \\ 1_jane@mail.ru \\ 1 Уфимский государственный нефтяной технический университет, \\ Россия, 450064, г. Уфра, ул. Космонавтов, 1.
}

\begin{abstract}
Актуальность. Разработана конструкция и принцип работы пневмокомпенсатора для плунжерного насоса с погружным магнитоэлектрическим двигателем, позволяющего снизить амплитуду колебаний давления на выкиде насоса путем выравнивания скорости потока жидкости в лифртовых трубах. При использовании в пневмокомпенсаторах диафрагмы, выполненной из резины с армированием, она становится устойчивой к разрушению, что в целом увеличивает эфрфективность работы и срок эксплуатации пневмокомпенсатора.

Цель: разработать пневмокомпенсатор для плунжерного насоса с погружным магнитоэлектрическим двигателем. Провести прочностной анализ и оценку эфффективности применения диафрагмы с армированием в пневмокомпенсаторах, применяемых для снижения пульсаций скорости и давления потока фрлюида в насосно-компрессорных трубах; обосновать выбор материала диафрагмы пневмокомпенсатора.

Объекты: скважинные пневмокомпенсаторы, погружной бесштанговый плунжерный насос с линейным магнитоэлектрическим двигателем, колонна насосно-компрессорных труб.

Методы: имитационное моделирование диафрагмы, закрепленной на перфорированной трубе, с применением программного комплекса «Компас 3D» модуль APMFEM; задание в граничных условиях расчетной модели технологических параметров при моделировании численных значений скорости, расхода, давления, температуры аналогичными действующей скважинной установке.

Результаты. Установлено, что максимальные напряжения, возникающие в диафрагме в процессе работы пневмокомпенсатора, в 4 раза меньше предельно допускаемых, что показывает работоспособность пневмокомпенсатора в скважинных условиях. Показано положительное влияние армирования диафррагмы с точки зрения снижения максимальных напряжений в эластичной оболочке. Проанализировано влияние технологических параметров пневмокомпенсаторов (суммарного газового объема, начального давления в газовой камере) на эфффективность их работы. Обосновано оптимальное давление зарядки пневмокомпенсаторов, которое не должно превышать минимально давление в насосно-компрессорных трубах в течение цикла откачки, чтобы исключить негативное влияние прижатия эластичной оболочки к перфорированной трубе (внутренней стенке пневмокомпенсатора).
\end{abstract}

\section{Ключевые слова:}

Пневматические компенсаторы давления, выравнивание пульсаций давления, диафррагма, установка плунжерного насоса с погружным линейным приводом, рабочая камера пневмокомпенсатора, прочностной анализ диафрагмы.

\section{Введение}

При добыче пластового флюида плунжерными насосами возникают пульсации давления жидкости в насосно-компрессорных трубах и выкидной линии скважин. Пульсационный характер давления возникает в результате воздействия циклических знакопеременных нагрузок, воспринимаемых погружным электродвигателем, на плунжер насоса. Вышесказанное повышает усталостный износ насосного оборудования и оказывает негативное влияние на надежность работы двигателя, энергопотребление насосной установки [1].

С целью выравнивания пульсаций скорости и давления при откачке жидкости плунжерными насосами и более эффективной добычи пластового флюида предложены пневматические компенсаторы давления (пневмокомпенсаторы), известные в нефтепромысловой практике для буровых насосов и штанговых установок. Работа пневмокомпенсаторов основана на по- нижении амплитуды колебаний давления за счет выравнивания скорости потока флюида в насоснокомпрессорных трубах (НКТ). Во время работы установки (рис. 1) при каждом цикле нагнетания происходит рост давления в полости НКТ, при этом часть откачиваемой скважинной жидкости через щелевые вырезы подается в рабочую камеру пневмокомпенсатора, сжимая газ в газовой камере, в которую предварительно закачан инертный газ под давлением. При снижении давления происходит вытеснение принятой жидкости в НКТ и возврат в исходное состояние диафрагмы за счет давления в газовой камере [2-4].

Пневмокомпенсатор состоит из муфты с обратным клапаном и сквозным цилиндрическим каналом, корпуса, трубы с щелевыми вырезами, нижней переводной муфты. Внутреннее пространство между корпусом и диафрагмой образует газовую камеру, а между трубой и диафрагмой - рабочую камеру. Пневмокомпенсатор устанавливается в нижней части НКТ на 
глубине порядка 1000 м. В расчетах полагается, что давление откачиваемой жидкости в месте установки пневмокомпенсатора выше давления насыщения нефти газом, что позволяет исключить влияние свободного газа, содержащегося в откачиваемой продукции, на его работу [5-7].

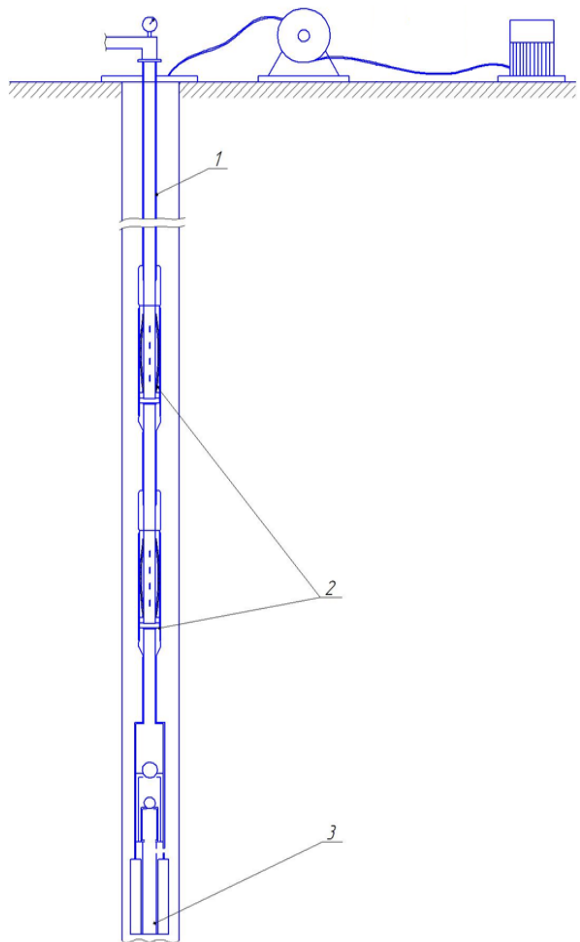

Pис. 1. Погружная бесштанговая насосная установка с пневмокомпенсаторами (1 - колонна НКТ, 2 пневмокомпенсаторы, 3 - погружной бесштанговый плунжерный насос)

Fig. 1. Submerged rodless pump installation with pneumatic compensators $(1$ - column of PCP (pump and compressor pipes), 2 - pneumatic compensators, 3 submerged rodless plunger pump)

В пневмокомпенсаторе для устранения пульсаций давления в колонне НКТ предлагается использовать резиновую диафрагму (рис. 1), которая производится компанией ООО «РЕАМ-РТИ».

Для сравнения рассматривали два типа резины:

1. Аф-15 (Aflas $100 \mathrm{H}$ ) резиновая смесь на основе фторэластомеров AFLAS (FEP, TFE/P) в соответствии с ТУ 2512-016-46521402-2004, предназначенная для формовых уплотнительных деталей подвижных и неподвижных соединений. РТИ (резинотехнические изделия) для УЭЦН (диафрагмы гидрозащиты, сильфоны торцевых уплотнений, уплотнительные кольца). Манжеты для пакеров и свабов обладают следующими свойствами:

- среда: минеральные и синтетические масла, пластовые жидкости, топлива, острый пар, меркаптан;

- температура: $-40 \ldots+250$, кратковременно до +300;

- свойства: стойкость к агрессивным средам, устойчивость к $\mathrm{H}_{2} \mathrm{~S}$ до $25 \%$, стойкость кессон- ная 1-2 класса по NACE TMO 192-98, устойчивость к воздействию пара, диэлектрические свойства, температура хрупкости $-48^{\circ} \mathrm{C}$.

2. PC-66 (Aflas/EPDM) резиновая смесь на основе фторэластомера и этиленпропиленового каучука (TFE/P|EPDM), предназначенная для формовых уплотнительных деталей подвижных и неподвижных соединений, РТИ для УЭЦН (диафрагмы гидрозащиты, уплотнительные кольца), обладающая следующими свойствами:

- среда: минеральные и синтетические масла, пластовые жидкости, топлива;

- температура, ${ }^{\circ} \mathrm{C}:-50 \ldots+230$;

- свойства: улучшенная морозостойкость и теплостойкость в сравнении с Аф-15.

C учетом проанализированных свойств материалов диафрагмы был выбран тип Аф-15, так как у данного типа резины более широкий диапазон применения в различных средах, в сравнении с резиной РС-66, а улучшенные морозостойкость и теплостойкость незначительное преимущество РС-66 перед Аф-15.

Диафрагма крепится двумя хомутами на двухдюймовой трубе, которая в зависимости от исполнения может быть выполнена с перфорацией отверстиями или щелевыми вырезами.

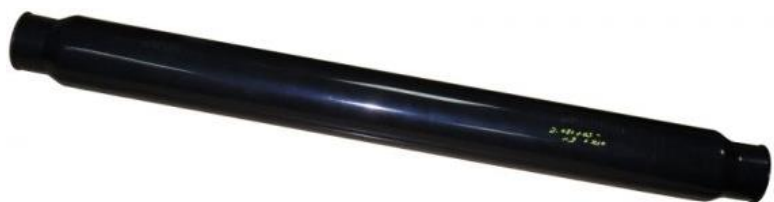

Pис. 2. Общий вид диафрагмы компенсатора

Fig. 2. General view of the compensator diaphragm

Внутри слоя резины расположены полиакрилнитрильные волокна типа «Орлон», которые выполнены в виде нитей и позволяют значительно повысить прочностные свойства резиновой смеси диафрагмы. Физические свойства материала диафрагмы показаны в табл. $1[8,9]$.

Таблица 1. Физические свойства материала диафрагмы Table 1. Physical properties of the diaphragm material

\begin{tabular}{|c|c|}
\hline \multicolumn{2}{|c|}{$\begin{array}{l}\text { Физические свойства волокна «Орлон» } \\
\text { Physical properties of «Orlon» fiber }\end{array}$} \\
\hline $\begin{array}{l}\text { Прочность на разрыв, кГс/см² } \\
\text { Tensile strength, } \mathrm{kGs} / \mathrm{sm}^{2}\end{array}$ & 3500 \\
\hline $\begin{array}{l}\text { Диаметр нити, мкм } \\
\text { Thread diameter, mkm }\end{array}$ & $10-25$ \\
\hline $\begin{array}{l}\text { Максимальная температура, }{ }^{\circ} \mathrm{C} \\
\text { Maximum temperature, }{ }^{\circ} \mathrm{C}\end{array}$ & 230 \\
\hline $\begin{array}{l}\text { Модуль упругости, } \mathrm{\kappa} \Gamma \mathrm{c} / \mathrm{cm}^{2} \\
\text { Elastic modulus, } \mathrm{kGs} / \mathrm{sm}^{2}\end{array}$ & $2,8 * 10^{3}$ \\
\hline \multicolumn{2}{|c|}{ Физические свойства резины/Physical properties of rubber } \\
\hline $\begin{array}{l}\text { Удлинение при разрыве, \% } \\
\text { Elongation at break, \% }\end{array}$ & $\begin{array}{c}\text { не менее } 100-150 \\
\text { not less than } 100-150\end{array}$ \\
\hline $\begin{array}{l}\text { Прочность при растяжении, кГс } / \mathrm{cm}^{2} \text {, } \\
\text { Tensile strength, } \mathrm{kGs} / \mathrm{sm}^{2}\end{array}$ & $\begin{array}{c}\text { не менее } 90-122 \\
\text { not less than } 90-122\end{array}$ \\
\hline $\begin{array}{l}\text { Предельная температура, }{ }^{\circ} \mathrm{C} \\
\text { Limiting temperature, }{ }^{\circ} \mathrm{C}\end{array}$ & 230 \\
\hline $\begin{array}{l}\text { Относительная остаточная деформация } \\
\text { после разрыва, \% } \\
\text { Relative permanent deformation after rup- } \\
\text { ture, \% }\end{array}$ & $\begin{array}{c}\text { не более 5-24 } \\
\text { no more than 5-24 }\end{array}$ \\
\hline
\end{tabular}


Таблица 2. Исходные данные

Table 2. Source data

\begin{tabular}{|c|c|}
\hline $\begin{array}{c}\text { Параметры } \\
\text { Parameters }\end{array}$ & $\begin{array}{l}\text { Значения } \\
\text { Values }\end{array}$ \\
\hline \begin{tabular}{|l} 
Длина хода плунжера, $S$, м \\
Stroke length of the plunger, $S, \mathrm{~m}$ \\
\end{tabular} & 1,2 \\
\hline $\begin{array}{l}\text { Число двойных ходов в минуту, } n \\
\text { Number of swings per minute, } n\end{array}$ & 10 \\
\hline Диаметр насоса, мм/Pump diameter, mm & 44 \\
\hline Глубина спуска насоса, м/Depth of pump descent, $\mathrm{m}$ & 1000 \\
\hline Дебит скважины, $Q$, т/сут/Well flow rate, $Q$, t/day & 20 \\
\hline Плотность нефти, $\rho_{\mathrm{H}}, \mathrm{\kappa r} / \mathrm{M}^{3} /$ Oil density, $\rho_{\mathrm{o}}, \mathrm{kg} / \mathrm{m}^{3}$ & 840 \\
\hline Плотность газа, $\rho_{г}, \mathrm{\kappa} \Gamma / \mathrm{m}^{3} / \mathrm{Gas}$ density, $\rho_{\mathrm{g}}, \mathrm{kg} / \mathrm{m}^{3}$ & 1,04 \\
\hline Плотность воды, $\rho_{\mathrm{B}}, \mathrm{kг} / \mathrm{M}^{3 /}$ Water density, $\rho_{\mathrm{w}}, \mathrm{kg} / \mathrm{m}^{3}$ & 1009 \\
\hline $\begin{array}{l}\text { Давление в газовой камере компенсатора, } P_{\mathrm{\kappa}}, \mathrm{MПа} \\
\text { Pressure in the gas chamber of the compensator, } P_{\mathrm{c}}, \mathrm{MPa}\end{array}$ & $9-10$ \\
\hline $\begin{array}{l}\text { Перепад давления при работе компенсатора, } \Delta P_{\text {пл }}, \mathrm{MПа} \\
\text { Pressure drop during compensator operation, } \Delta P_{\mathrm{p}}, \mathrm{MPa}\end{array}$ & $0,1-0,4$ \\
\hline Диаметр НКТ, $d_{\mathrm{H}}$, Дюймы/Tubing diameter, $d_{\mathrm{n}}$, inches & 2 \\
\hline $\begin{array}{l}\text { Наружный диаметр диафрагмы, мм } \\
\text { Outer diaphragm diameter, mm }\end{array}$ & 88,6 \\
\hline \begin{tabular}{|l} 
Толщина стенки диафрагмы, мм \\
Diaphragm wall thickness, mm
\end{tabular} & 5 \\
\hline \begin{tabular}{|l|} 
Площадь одного выреза (14 вырезов) трубы \\
с щелевыми вырезами, мм² \\
Area of one cutout (14 cutouts) of slotted pipes, $\mathrm{mm}^{2}$
\end{tabular} & 50 \\
\hline $\begin{array}{l}\text { Объем жидкости, вмещаемый компенсатором, л } \\
\text { Liquid volume, accommodated by the expansion joint, } 1\end{array}$ & 2 \\
\hline
\end{tabular}

Наиболее важным требованием, предъявляемым к резинам, используемым в комплектующих для нефтедобывающего оборудования, является их стойкость к условиям, при которых возникают большие рабочие давления, а также изменяется давление окружающей среды, т. е. так называемая «кессонная» стойкость.

При изготовлении диафрагмы компенсатора применятся резиновая смесь марки Aф (Aflas (тетрафторэтиленпропилен TFE/P (FEPM)). Это резина, не содержащая серу, рабочая температура до $230{ }^{\circ} \mathrm{C}$. Среды, в которых эксплуатируется резина: минеральное и МДПН масло, синтетические масла ShellFluid 4600, топлива, спирты, бензин, кислоты и щелочи, водяной пар, пластовая жидкость [10-12].

На первом этапе выполнено моделирование гидродинамических процессов в НКТ при работе пнев-

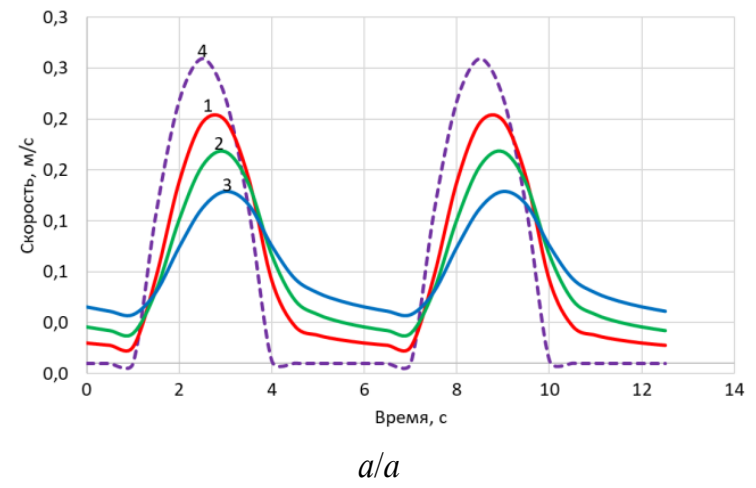

мокомпенсаторов, основанное на численном решении уравнений Навье-Стокса [13]. Параметры технологического режима эксплуатации насосной установки и пневмокомпенсаторов представлены в табл. 2.

Проанализировано влияние технологических параметров пневмокомпенсаторов (газовый объем, начальное давление в газовой камере - давление зарядки) на эффективность работы пневмокомпенсаторов. Результирующий газовый объем регулируется изменением количества пневмокомпенсаторов, устанавливаемых в скважине. С увеличением суммарного газового объема пневмокомпенсаторов амплитуда колебаний скорости потока и давления в НКТ закономерно снижаются (рис. 3). В частности, при увеличении результирующего объема газовых камер от 25 до 100 л амплитуда колебаний давления снижается от 0,42 до 0,15 МПа. С увеличением суммарного количества и соответственно газового объема пневмокомпенсаторов не только снижается амплитуда колебаний давления, но также и уменьшается рабочий ход (т. е. амплитуда деформаций) диафрагмы для отдельно взятого пневмокомпенсатора, что способствует снижению темпов усталостного износа и увеличению срока эксплуатации (долговечности) диафрагмы.

Следует отметить также, что в рассмотренном примере цикловая подача насоса составляет около 1,8 л, при этом суммарный газовый объем системы пневмокомпенсаторов, необходимый для эффективного сглаживания пульсаций давления (в частности, для кривых 2, 3 на рис. 3 он составляет 50 и 100 л соответственно), кратно превышает величину цикловой подачи. Вышесказанное связано с тем, что изменение объема газовых камер, соответствующее объему принимаемой жидкости, связано с изменением давления в НКТ и ограничено (в процессе сжатия газовой камеры и приема жидкости давление в газовой камере возрастает, и, если оно достигает давления в НКТ, прием жидкости прекращается). В результате при работе пневмокомпенсатора полезной (т. е. принимающей жидкость) является только часть объема газовой камеры.

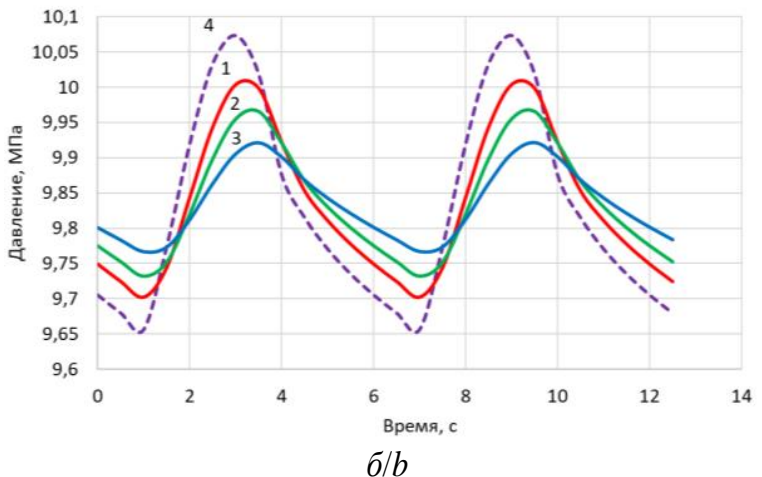

Рис. 3. Динамика скорости потока (а) и давления (б) в нижнем коние НКТ при различном суммарном газовом объеме системы пневмокомпенсаторов (1 - 25 л; 2 - 50 л; 3 - 100 л; 4 - без пневмокомпенсаторов)

Fig. 3. Dynamics of the flow rate (a) and pressure $(b)$ at the lower end of the tubing with different total gas volume of the pneumatic compensator system $(1-25 l ; 2-50 l ; 3-100 l ; 4$-without pneumatic compensators) 
Таблица 3. Параметры и результаты разбиения

Table 3. Parameters and results of splitting figure

\begin{tabular}{|l|c|}
\hline \multicolumn{1}{|c|}{$\begin{array}{c}\text { Наименование } \\
\text { Name }\end{array}$} & $\begin{array}{c}\text { Значение } \\
\text { Values }\end{array}$ \\
\hline Длина стороны ячейки, мм/Cell side length, mm & 5 \\
\hline $\begin{array}{l}\text { Коэффициент разрежения в объеме } \\
\text { Volume dilution ratio }\end{array}$ & 1,5 \\
\hline $\begin{array}{l}\text { Максимальный коэффициент сгущения } \\
\text { на поверхности } \\
\text { Махітит surface thickening factor }\end{array}$ & 1 \\
\hline Число конечных элементов/Finite element count & 49949 \\
\hline Количество узлов/Number of nodes & 16463 \\
\hline
\end{tabular}

На втором этапе выполнен прочностной анализ с применением программного комплекса «Компас 3D» модуль APMFEM. Перед началом прочностного анализа в программном комплексе «Компас 3D» модуль APMFEM 3D модель диафрагмы разбивается на конечно-элементную сетку (рис. 4), параметры которой показаны в табл. 3. Далее были приложены нагрузки, соответствующие рабочему режиму эксплуатации компенсатора, указанные в табл. 2, задан материал исполнения диафрагмы (с армированием и без) и проведен статический расчет [14-16]. На рис. 5 показана модель распределения максимальной величины напряжений в диафрагме.

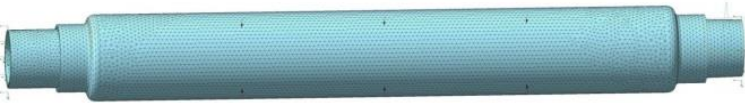

Pис. 4. Модель диафрагмы, закрепленной на перфорированной трубе, разбитая на конечно-элементную сетку в программном комплексе «Компас 3D» модуль АРMFEM

Fig. 4. Model of the diaphragm fixed to a perforated pipe, divided into a finite element grid in the software package «Compass 3D» module APMFEM

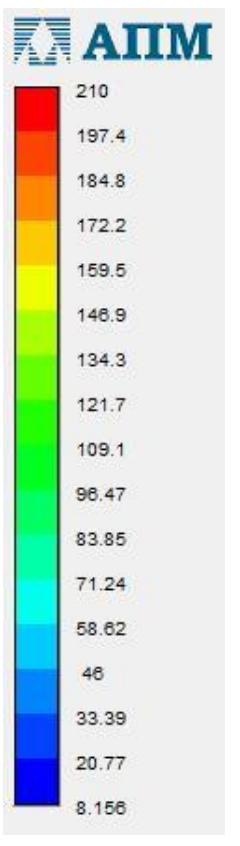

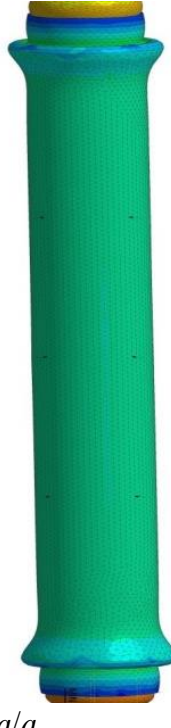

$a$
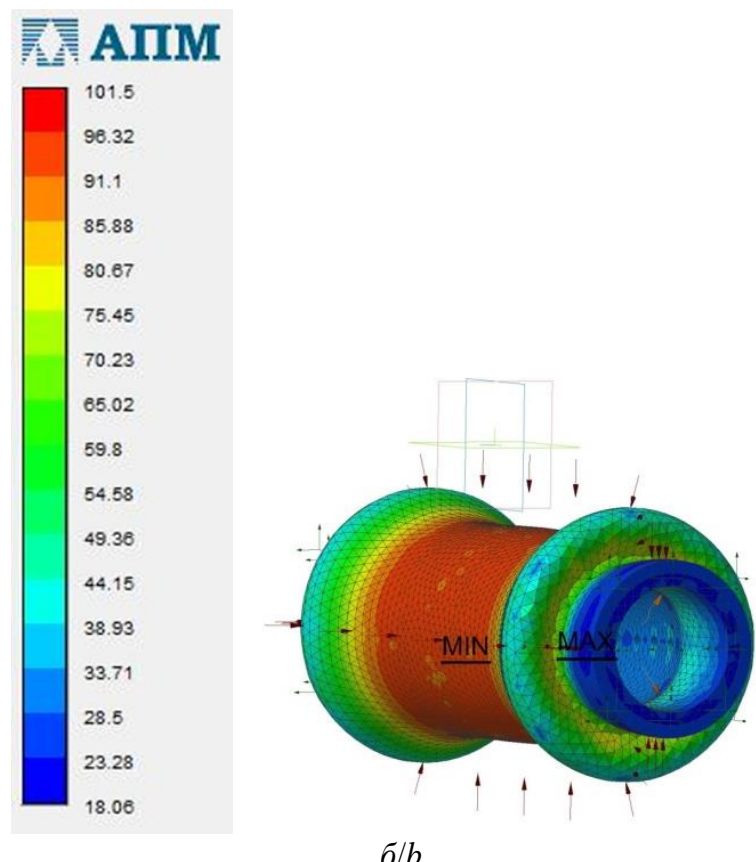

$\sigma / b$

Pис. 5. Сравнение распределения напряжений МПа в диафрагме (а - диафрагма с армированием, б - диафрагма без армирования) в программном комплексе «Компас 3D» модуль АРMFEM

Fig. 5. Comparison of the voltage distribution of MPa in the diaphragm ( $a$-diaphragm with reinforcement, $b-$ diaphragm without reinforcement) in the software package «Compass 3D» module APMFEM

Одной из основных эксплуатационных характеристик является прочность диафрагмы на разрыв, которая по данным изготовителя составляет 340 МПа для резины типа АФ-15 с армированием. Из графиков видно, что максимальные напряжения, возникающие в диафрагме в процессе работы пневмокомпенсатора, составляют порядка 85 МПа, что в 4 раза меньше предельно допускаемых. Максимальные напряжения для диафрагмы без армирования несколько выше (на $11 \%$, что составляет около 95 МПа), поскольку при растяжении стенок диафрагмы часть нагрузки воспринимает армирующая сетка. Кроме того, следует отметить, что в армированной диафрагме не возникает резких перепадов напряжений во всем сечении, т. е. материал менее подвержен появлению мелких трещин в тех местах, где возникают цикличные нагрузки.

Важное влияние на эффективность и надежность работы пневмокомпенсатора оказывает давление зарядки (давление предварительно закачиваемого газа на устье скважины). На графиках (рис. 6) показано влияние давления зарядки на величину колебаний давления в НКТ погружной установки с пневмокомпенсаторами (суммарный газовый объем в расчетах принят 50 л). Видно, что при равном суммарном газовом объеме пневмокомпенсаторов увеличение давления зарядки с 9 до 10 МПа приводит к снижению эффективности сглаживания колебаний скорости потока и давления в НКТ, поскольку давление зарядки 10 
МПа является чрезмерно высоким: при снижении давления в НКТ до величины, меньше давления зарядки, в процессе расширения газовой камеры диафрагма полностью прилегает к перфорированной трубе, что обусловлено его конструктивными особенностями, и подача пневмокомпенсатора прекращается (т. е. дальнейшее расширение газовой камеры ограничено конструктивными особенностями пневмокомпенсатора), нарушая его нормальную работу. Негативное влияние высокого давления зарядки также обусловлено следующим: если в перфорированной трубе давление меньше, чем в газовой полости компенсатора и диафрагма прижата давлением газа к перфорированной трубе, тогда давление газа в местах расположения от-

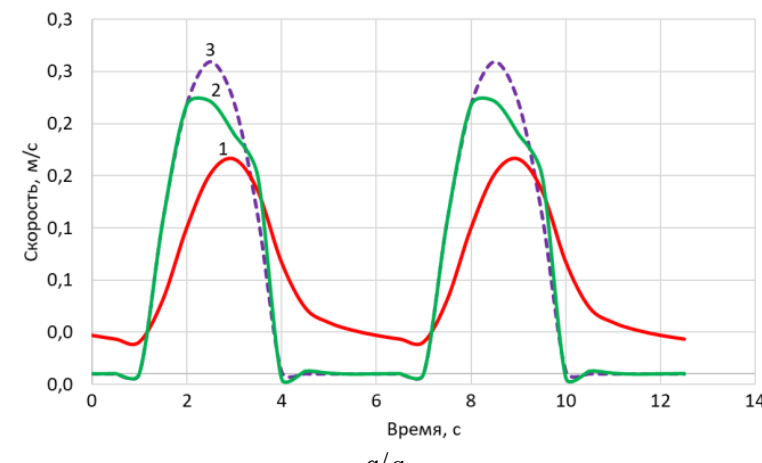

$a / a$ верстий выдавливает эластичную оболочку в отверстия. В этом случае армирование стенки оказывает положительное влияние на надежность работы диафрагмы, сводя к минимуму эффект выдавливания эластичной оболочки [17-20]. Таким образом, оптимальное давление зарядки не должно превышать минимальное давление в НКТ в течение цикла откачки, чтобы исключить негативное влияние прижатия эластичной оболочки к перфорированной трубе. Кроме того, необходимо учитывать и тот факт, что с ростом глубины установки пневмокомпенсаторов увеличивается естественная геотермическая температура и давление в газовой полости пневмокомпенсаторов возрастает относительно устьевой величины.

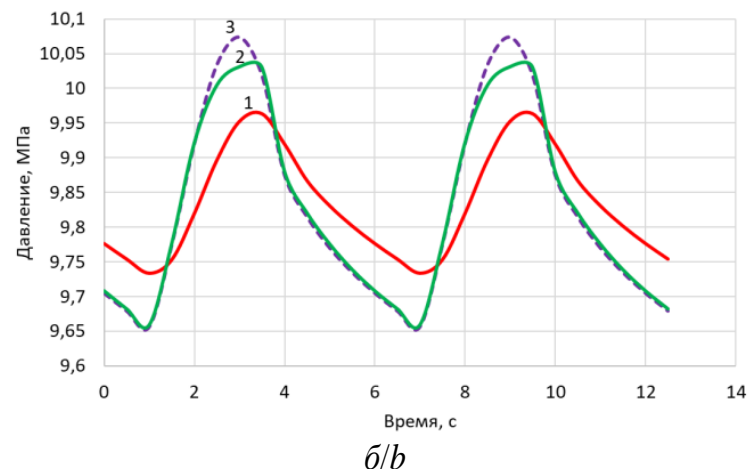

Рис. 6. Динамика скорости потока (а) и давления (б) в нижнем конще НКТ при различном давлении зарядки (1 8 МПа; 2 - 10 МПа; 3 - без пневмокомпенсаторов)

Fig. 6. Dynamics of the flow rate (a) and pressure (b) at the lower end of PCP at different charging pressures $(1-8 M P a$; 2 - $10 \mathrm{MPa} ; 3$ - without pneumatic compensators)

Достоинством разработанного пневмокомпенсатора является то, что его применение позволяет улучшить технологический режим работы скважинной безштанговой насосной установки и снизить колебания давления и скорости потока в НКТ. Для эффективной и надежной работы необходимо согласование рабочих параметров компенсаторов с технологическими параметрами насосной установки (цикловой подачей, глубиной спуска насоса и др.).

\section{Выводы}

1. Разработана конструкция, и показан принцип работы пневмокомпенсатора для установки плунжерного насоса с погружным линейным приводом. Построена имитационная модель работы резиновой диафрагмы в пневмокомпенсаторе. Установлено, что максимальные напряжения, возникающие в диафрагме в процессе работы пневмокомпенсатора, в 4 раза меньше предельно допускаемых, что показывает работоспособность пневмокомпенсатора в скважинных условиях. Максимальные напряжения для диафрагмы без армирования несколько выше, чем для диафрагмы с ар-

\section{СПИСОК ЛИТЕРАТУРЫ}

1. Николаев Г.И., Уразаков К.Р., Валеев М.Д. Совершенствование эксплуатации наклонных и обводнившихся глубиннонасосных скважин // Нефтяное хозяйство. - 1980. - № 1. - С. 32-35. мированием (на 11 \%), поскольку при растяжении стенок диафрагмы часть нагрузки воспринимает армирующая сетка.

2. Проанализировано влияние технологических параметров пневмокомпенсаторов (суммарного газового объема, начального давления в газовой камере) на эффективность работы пневмокомпенсаторов. Показано, что с увеличением суммарного количества и соответственно газового объема пневмокомпенсаторов снижается амплитуда колебаний давления (в модельном примере в 3 раза), а также амплитуда деформаций диафрагмы для каждого пневмокомпенсатора, что способствует снижению темпов усталостного износа и увеличению срока эксплуатации (долговечности) диафрагмы. Обосновано оптимальное давление зарядки пневмокомпенсаторов, которое не должно превышать минимальное давление в НКТ в течение цикла откачки, чтобы исключить негативное влияние прижатия эластичной оболочки к перфорированной трубе (внутренней стенке пневмокомпенсатора).

2. Зотов А.Н., Тимашев Э.О., Уразаков К.Р. Методы гашения колебаний давления на устье штанговых установок // Нефтегазовое дело. - 2018. - Т. 16. - № 6. - С. 56-64.

3. Уразаков К.Р., Тимашев Э.О., Тухватуллин Р.С. Устьевой пневмокомпенсатор штанговой скважинной насосной установки // Территория «НЕФТЕГАЗ». - 2017. - № 12. - С. 60-64. 
4. Нигматулин Р.И. Динамика многофазных сред. - М.: Изд-во «Наука», 1987. - 464 с.

5. Гидродинамика скважинного гидромеханического компенсатора колебаний давлений промывочной жидкости / М.С. Габдрахимов, Ф.И. Ермоленко, Т.Н. Миннивалеев, Р.И. Сулейманов // Экспозиция нефть и газ. - 2015. - № 6. - С. 14-16.

6. Байбурин И.Р., Булюкова Ф.З., Ямалиев В.У. Особенности эксплуатации УЭЦН в осложненных условиях ООО РНСтавропльнефтегаз // Нефтегазовое дело. - 2011. -Т. 9. № 1. - C. 31-34.

7. SolidWorks Flow Simulation 2012 Tutorial // Docslide. 2014 URL: https://www.solidworks.com/sw/docs/edu_simulation_hotd instructor 2012.pdf (дата обращения 11.06.2020).

8. Бахтияров Р.Х. Осложнения при эксплуатации скважин УЭЦН и методы повышения эффективности эксплуатации УЭЦН // Современные технологии в нефтегазовом деле-2019: Международная научно-техническая конференция. - Уфа: Уфимский государственный нефтяной технический университет, 2019. С. 28-32.

9. Романова Н.А. Повышение эффективности работы штанговых насосных установок при добыче высоковязких нефтей: автореф. дис. ... канд. техн. наук. - Уфа, 2010. - 23 с.

10. Скважинный компенсатор гидроудара: пат. Рос. Федерация, № 112261 заявл. 25.02.2011; опубл. 10.01.2012. Бюл. № 1. - 5 с

11. Скважинный компенсатор давления: пат. Рос. Федерация, № 2591235 заявл. 10.05.2015; опубл. 20.07.2016. Бюл. № 20. $15 \mathrm{c}$.

12. Research on intelligent power supply control based on sensor-less temperature identification of Electric Submersible Motor / H. Zhang, J. Yu, Q. Jiang, L. Wang, D. Xu // Green World with Power Electronics: 9th International Conference on Power Electronics - ECCE Asia. - USA, 2015. - P. 2807-2808.

13. Perforating the largest deepwater wells in Brazil - minimizing shock loads / C.E. Baumann, R.P. Scudino, M.E. Smart,
M.J. Tsuchie, E. Schnitzler, R.S. Roman // Offshore Technology Conference. - Brazil, 2019. - V. 22. - P. 88-90.

14. Baumann C.E., Oden J.T. An adaptive-order discontinuous Galerkin method for the solution of the Euler equations of gas dynamics // International Journal for Numerical Methods in Engineering. - 2000. - V. 47 (1-3). - P. 61-73.

15. Perforating high-pressure deepwater wells in the Gulf of Mexico / C. Baumann, H. Williams, T. Korf, R. Pourciau // SPE Annual Technical Conference and Exhibition. - Denver, CO, United States, 2011. - V 4. - P. 2744-2756.

16. Pressure dependent permeability: unconventional approach on well performance / T.C. Nguyen, S. Pande, D. Bui, E. Al-Safran, H.V. Nguyen // Journal of Petroleum Science and Engineering. 2020. - V. 193. - 107358.

17. El-Saghier R.M., Abu El Ela M., El-Banbi A. A model for calculating bottom-hole pressure from simple surface data in pumped wells // Journal of Petroleum Exploration and Production Technology. - 2020. - V. 10 (5). - P. 2069-2077.

18. Dynamics and frequency and voltage control of downhole oil pumping system / W. Li, V. Vaziri, S.S. Aphale, S. Dong, M. Wiercigroch // Mechanical Systems and Signal Processing. 2020. - V. 139. - 106562.

19. Improved method on hydraulic power calculations for conventional sucker rod pumping system / O.E. Mwangupili, P. Chunsheng, C. Bululu, S.L. Nyanswi, S.M. Muya // IOP Conference Series: Earth and Environmental Science. - 2019. V. 431. -012026 .

20. Тимашев Э.О., Уразаков К.Р. Обоснование снижения нагрузок на привод погружных плунжерных установок, оборудованных пневмокомпенсаторами // Нефтяное хозяйство. - 2020. V. 5. - P. 88-92.

Поступила: 30.06 .20202$.

\section{Информация об авторах}

Уразаков К.P., доктор технических наук, профессор кафедры машин и оборудования нефтегазовых промыслов, Уфимский государственный нефтяной технический университет.

Tимашев Э.О., кандидат технических наук, докторант кафедры машин и оборудования нефтегазовых промыслов, Уфимский государственный нефтяной технический университет.

Абдуллин Н.A., кандидат технических наук, доцент кафедры пожарной и промышленной безопасности, Уфимский государственный нефтяной технический университет. 
UDC 622.276.53.054

\title{
PNEUMATIC COMPENSATOR FOR PLUNGER PUMP WITH SUBMERSIBLE LINEAR DRIVE
}

\author{
Kamil R. Urazakov ${ }^{1}$, \\ urazakk@mail.ru \\ Eduard 0. Timashev ${ }^{1}$, \\ timashev@mail.ru \\ Nail A. Abdullin 1 , \\ 1_jane@mail.ru \\ 1 Ufa State Petroleum Technological University, \\ 1, Kosmonavtov street, Ufa, 450064, Russia.
}

\begin{abstract}
The relevance. The authors have developed the design and operation principle of a pneumatic compensator for a plunger pump with a submerged magnetoelectric motor, which allows reducing the amplitude of pressure fluctuations at the pump outlet by equalizing liquid flow rate in the lift pipes. It is shown that when using a diaphragm made of reinforced rubber in pneumatic compensators, it becomes resistant to destruction, which in general increases the efficiency and service life of the pneumatic compensator.

The main aim of the research is to develop a pneumatic compensator for a plunger pump with a submerged magnetoelectric motor; conduct a strength analysis and evaluation of the effectiveness of the diaphragm with reinforcement in pneumatic compensators, which used to reduce the pulsations of the speed and pressure of the fluid flow in the pump and compressor pipes; justify the choice of the diaphragm material of the pneumatic compensator.

Objects: downhole pneumatic compensators, submerged rodless plunger pump with linear magnetoelectric motor, column of pump and compressor pipes.

Methods: simulation of the diaphragm fixed on a perforated pipe using the software package "Compass 3D» module of the APMFEM; setting the design model of technological parameters in boundary conditions of the design model of technological parameters, at modeling numerical values of speed, flow rate, pressure, temperature similar to the existing well installation.

Results. It is established that the maximum stresses that occur in the diaphragm during the operation of the pneumatic compensators are 4 times less than the maximum permissible ones, which shows the operability of the pneumatic compensator in downhole conditions. The positive effect of diaphragm reinforcement in terms of reducing the maximum stresses in the elastic shell is shown. The influence of technological parameters of pneumatic compensators (total gas volume, initial pressure in the gas chamber) on the efficiency of pneumatic compensators is analyzed. The optimal charging pressure of pneumatic compensators is justified, which should not exceed the minimum pressure in the tubing during the pumping cycle, in order to exclude the negative impact of pressing the elastic shell against the perforated pipe (the inner wall of the pneumatic compensator).
\end{abstract}

\section{Key words:}

Pneumatic pressure compensators, pressure pulsation equalization, diaphragm, installation of a plunger pump with a submersible linear drive, working chamber of pneumatic compensator, diaphragm strength analysis.

\section{REFERENCES}

1. Nikolaev G.I., Urzakov K.R., Valeev M.D. Sovershenstvovanie ekspluatatsii naklonykh i obvodnivshikhsya glubinnonasosnykh skvazhin [Improving the operation of inclined and flooded deeppump wells]. Oil economy, 1980, no. 1, pp. 32-35.

2. Zotov A.N., Timaschev E.O., Urzakov K.R. Methods of damping pressure fluctuations at the mouth of rod installations. Oil and Gas industry, 2018, vol. 16, no. 6, pp. 56-64. In Rus.

3. Urzakov K.R., Timaschev E.O., Tukhvatullin R.S. Wellhead pneumatic compensator of a rod well pumping unit. Territory «NEFTEGAZ», 2017, no. 12, pp. 60-64. In Rus.

4. Nigmatullin R.I. Dinamika mnogofaznykh sred [Dynamics of multiphase media]. Moscow, Nauka Publ., 1987. 464 p.

5. Gabdrakhimov M.S., Ermolenko F.I., Minnivaleev T.N., Suleymanov R.I. Hydrodynamics of a borehole hydro-mechanical compensator for pressure fluctuations of washing liquid. Oil and gas exposition, 2015, no. 6, pp. 14-16. In Rus.

6. Baiburin I.R., Bulykov F.Z., Yamaleev V.W. Features of IECP operation (installation of an electric centrifugal pump) in complicated conditions LLC RN-Stavropolneftegaz. Oil and gas, 2011, vol. 9, no. 1, pp. 31-34. In Rus.

7. SolidWorks Flow Simulation 2012 Tutorial. Docslide, 2014. Available at: https://www.solidworks.com/sw/docs/edu_simulation hotd_instructor_2012.pdf (accessed 11 June 2020).

8. Bakhtiyarov R.Kh. Oslozhneniya pri ekspluatatsii skvazhin UECN i metody povyshehiya effektivnosti ekspluatatsii UECN [Complications in the operation of wells IECP (installation of an electric centrifugal pump) and methods for exploitation improving IECP's efficiency]. Sovremennye tekhnologii v neftegazovom dele 2019. Mezhdunarodnaya nauchno-tekhnicheskaya konferentsiya [Modern technologies in the oil and gas industry. International scientific and technical conference]. Ufa, Ufa State Oil Technical University, 2019. pp. 28-32.

9. Romanova N.A. Povyshenie effektivnosti raboty shtangovykh nasosnykh ustanovok pri dobyche vysokovyazkikh neftey. Avtoreferat Dis. Kand. nauk [Improving the efficiency of rod pumping units in production of high-viscosity oils. Cand. Diss. Abstract]. Ufa, 2010. $23 \mathrm{p}$.

10. Eliseev A.D., Maltsev P.V., Neskromnykh V.V. Skvazhinny kompesator gidroudara [Downhole hydraulic shock compensator]. Patent RF, no. 112261, 2012.

11. Hallunbek I. Skvazhinny kompesator davleniya [Downhole pressure compensator]. Patent RF, no. 2591235, 2016.

12. Zhang H., Yu J., Jiang Q., Wang L., Xu D. Research on intelligent power supply control based on sensor-less temperature identification of Electric Submersible Motor. $9^{\text {th }}$ International Conference on Power Electronics, ECCE Asia. Green World with Power Electronics. USA, 2015. pp. 2807-2808.

13. Baumann C.E., Scudino R.P., Smart M.E., Tsuchie M.J., Schnitzler E., Roman R.S. Perforating the largest deepwater wells in Brazil. Minimizing shock loads. Offshore Technology Conference. Brazil, 2019. Vol. 22, pp. 88-90.

14. Baumann C.E., Oden J.T. An adaptive-order discontinuous Galerkin method for the solution of the Euler equations of gas dynamics. International Journal for Numerical Methods in Engineering, 2000, vol. 47, pp. 61-73. 
15. Baumann C., Williams H., Korf T., Pourciau R. Perforating highpressure deepwater wells in the Gulf of Mexico. SPE Annual Technical Conference and Exhibition. Denver, CO, United States, 2011. Vol. 4, pp. 2744-2756.

16. Nguyen T.C., Pande S., Bui D., Al-Safran E., Nguyen H.V. Pressure dependent permeability: Unconventional approach on well performance. Journal of Petroleum Science and Engineering, 2020 vol. 193, Article number 107358

17. El-Saghier R.M., Abu El Ela M., El-Banbi A. A model for calculating bottom-hole pressure from simple surface data in pumped wells. Journal of Petroleum Exploration and Production Technology, 2020, vol. 10 (5), pp. 2069-2077.

18. Li W., Vaziri V., Aphale S.S., Dong S., Wiercigroch M. Dynamics and frequency and voltage control of downhole oil pumping sys- tem. Mechanical Systems and Signal Processing, 2020, vol. 139, Article number 106562.

19. Mwangupili O.E., Chunsheng P., Bululu C., Nyanswi S.L., Muya S.M. Improved method on hydraulic power calculations for conventional sucker rod pumping system. IOP Conference Series: Earth and Environmental Science, 2019, vol. 431, Article number 012026.

20. Timashev E.O., Urazakov K.R. Obosnovanie snizheniya nagruzok na privod pogruzhnykh plunzhernykh ustanovok, oborudovannykh pnevmokompensatorami [Justification of reducing loads on the drive of downhole plunger installations with pneumatic compensators]. Oil Industry, 2020, vol. 5, pp. 88-92.

Received: 30 June 2020.

\section{Information about the authors}

Kamil R. Urazakov, Dr. Sc., professor, Ufa State Petroleum Technological University.

Eduard O.Timashev, doctoral candidate, Ufa State Petroleum Technological University.

Nail A. Abdullin, Cand. Sc., associate professor, Ufa State Petroleum Technological University. 